\title{
Copycat dynamics in leaderless animal group navigation
}

\author{
Edward A Codling* and Nikolai WF Bode
}

\begin{abstract}
Background: Many animals are known to have improved navigational efficiency when moving together as a social group. One potential mechanism for social group navigation is known as the 'many wrongs principle', where information from many inaccurate compasses is pooled across the group. In order to understand how animal groups may use the many wrongs principle to navigate, it is important to consider how directional information is transferred and shared within the group.

Methods: Here we use an individual-based model to explore the information-sharing and copying dynamics of a leaderless animal group navigating towards a target in a virtual environment. We assume that communication and information-sharing is indirect and arises through individuals partially copying the movement direction of their neighbours and weighting this information relative to their individual navigational knowledge.

Results: We find that the best group navigation performance occurs when individuals directly copy the direction of movement of a subset of their neighbours while only giving a small (6\%) weighting to their individual navigational knowledge. Surprisingly, such a strategy is shown to be highly efficient regardless of the level of individual navigational error. We find there is little relative improvement in navigational efficiency when individuals copy from more than 7 influential neighbours.

Conclusions: Our findings suggest that we would expect navigating group-living animals to predominantly copy the movement of others rather than relying on their own navigational knowledge. We discuss our results in the context of individual and group navigation behaviour in animals.
\end{abstract}

Keywords: Many wrongs principle, Collective behaviour, Animal movement, Navigation, Animal group, Individual-based model

\section{Background}

Many animal species are highly social and belonging to a group is known to have a number of benefits such as predator avoidance, foraging, mating, etc. [1]. Group membership may also improve consensus decision-making [2-4] and can provide navigational benefits to animals [5-8]. Here, we investigate group navigation, the ability of animal groups to direct their movement towards a spatial target (e.g. a source of food or a roosting site [5-8]), a process known as 'taxis' if it is based on local environmental cues [9]. At the level of the group, 'collective taxis' does not necessarily require individual level taxis to be present. For example, [10] demonstrated how a combination of individual kinesis (an undirected movement response to a stimulus)

\footnotetext{
* Correspondence: ecodling@essex.ac.uk

Department of Mathematical Sciences, University of Essex, Colchester, UK
}

and group cohesion results in taxis at the group level. However, many animal species are known to perform taxis at the individual level [9]. In this context, improved group navigation can be explained by mechanisms ranging along a continuum of shared decision-making mechanisms. At one end of this continuum, decisions may not be shared and group navigation can be explained by the 'leader-follower' model, where individuals with little or no navigational knowledge follow leaders (who have good or perfect directional information), and the group is able to make decisions and navigate efficiently [6,11-13]. In such cases, individuals with little or no navigational knowledge perform improved navigation by following leaders. At the other end of the continuum, decisions can be considered to be shared evenly between group members since all individuals in the 
group have equally poor navigation abilities and there are no leaders (the 'many wrongs principle'; [5]). In this case, the group navigation performance is improved through 'the pooling of many inaccurate compasses', while group cohesion acts to suppress navigation errors [5]. Findings in various empirical studies on navigating animal groups such as migrating birds [14,15], and humans [16], match the predictions of the many wrongs principle and it has been hypothesised as a mechanism used by larval reef fish navigating and recruiting to suitable coral reef habitats $[17,18]$. It should be noted that it is of course possible that leaders in the 'leader-follower' model could also benefit from pooling and sharing of navigational information. However, similar to [7], in this study we consider a homogenous group where individuals have equal navigational ability and there are no 'leaders'.

Simulations can be a useful exploratory tool to study the many wrongs hypothesis of animal group navigation in various contexts $[19,20]$. For example, Hancock et al. [21] considered a localised search problem and explored how the many wrongs principle might evolve in a population of foraging mammals. In the context of a global navigation problem, Grünbaum developed an individualbased model for taxis in a noisy environment based on turning rates [19]. A key finding of Grünbaum's study was that, in certain situations, high alignment turning rates (corresponding to stronger social interactions) made groups less flexible by prolonging group movement directions that had become erroneous [19]. The fact that stronger social interactions may not always be beneficial for group navigation is a concept that we will revisit in our simulation results. Guttal \& Couzin [22] and Torney et al. [23] used simulations to conceptually demonstrate how both the 'leader-follower' and the 'many-wrongs' model for group navigation can evolve in animal populations where individual fitness is obtained by balancing navigation success against costs of investment into navigation or social abilities. Codling et al. [7] showed that the many wrongs principle can be a successful strategy for homogeneous animal groups navigating towards a fixed target. Specifically, in [7] the authors considered a model where individuals navigate by partially copying the movement direction of their nearest neighbours and equally weighting this information relative to their own individual navigational knowledge. The choice of an equal weighting between these two sources of directional information was arbitrary and a systematic study of the effect of using different weightings on the group navigation efficiency was not undertaken [7]. However, earlier work on individual navigation (taxis) by Benhamou \& Bovet [24] demonstrated that the optimal weighting between a tactic component (navigation) and persistence (the tendency to continue moving in the same direction) is as low as $10 \%$ taxis, and this holds for all levels of individual navigation error. In addition, various studies have shown that there may be an optimal number of influential neighbours that contribute to information sharing [25-28].

In this paper we extend the simulation model used in [7] and explore the information-sharing and copying dynamics of a leaderless social animal group navigating towards a fixed circular target in a virtual environment. We assume that communication and information-sharing is indirect and arises through individuals partially copying the movement direction of their neighbours and weighting this information relative to their individual navigational knowledge. To assess how well simulated groups are able to navigate towards the target, we compute the navigational efficiency, which is inspired by and follows the terminology of previously developed metrics for the navigation performance of individual-level movement towards a target [24]. The navigational efficiency ranges in value from 1 (movement in a straight line directly towards the target), through 0 (no net movement towards or away from the target), to -1 (movement in a straight line directly away from the target). We find that the strategy that gives the best group navigation performance is when individuals directly copy the movement direction of their nearest neighbours while only giving a small (c6\%) weighting to their individual navigational knowledge. Navigation performance improves asymptotically with the number of influential neighbours, and there is little relative navigational gain by copying more than 7 neighbours. This navigation strategy is shown to perform well regardless of the level of individual navigational error.

\section{Results}

As shown in Table 1, we consider a range of values for the weighting given to individual navigation, $w$, the number of influential neighbours, $k$, and the standard deviation of the individual navigation error, $\varepsilon$. We compared all combinations of these parameters systematically and all other parameters in the simulation remain fixed throughout. For each parameter combination tested, we ran 100 replicate simulations.

\section{Effect of individual navigation weighting and number of influential neighbours}

In our simulation model, $w=0$ corresponds to undirected group movement where there is no navigational element to the movement of any individual (essentially the group moves as an undirected random walk [29]). In this case the mean navigational efficiency, $E$, is expected to be below zero, since when moving entirely randomly with no navigation in a two-dimensional environment, it is more likely that the group will move further away from the target than closer to it. This result is indeed found in our simulation results (Figure 1) for all levels of 
Table 1 Simulation model parameter descriptions and values

\begin{tabular}{|c|c|c|c|}
\hline $\begin{array}{l}\text { Model } \\
\text { parameter }\end{array}$ & Description & Fixed value & Range of values \\
\hline N & Total group size (individuals) & 40 & $2,5,10,15,20,25,30,40,50,60,80,100$ \\
\hline$R_{C}$ & Radius of collision avoidance & 2 & - \\
\hline$R_{0}$ & Radius of orientation & 15 & - \\
\hline$\xi$ & $\begin{array}{c}\text { Standard deviation of added environmental } \\
\text { noise/error }\end{array}$ & 0.1 & - \\
\hline$w$ & Weighting towards individual navigation (tactic factor) & - & $0.01,0.02,0.04,0.06,0.08,0.1,0.12,0.15,0.2,0.25,0.3,0.4,0.5,0.7,1$ \\
\hline k & Number of influential nearest neighbours & - & $1,2,3,4,5,7,9,12,15$ \\
\hline$\varepsilon$ & Standard deviation of individual navigation error & - & $0,0.2,0.5,1,1.5,2,3,5$ \\
\hline
\end{tabular}

Parameters have either no units or arbitrary distance units.

Fixed parameters are the same throughout all simulations. Parameters that have a range of values are explored in simulations using each combination of values in turn.

the individual navigation error, $\varepsilon$, and number of influential neighbours, $k$. Note that in the simulation results we are measuring mean average efficiency and it is possible for individual realisations of the simulation to finish with a positive (or negative) efficiency where the group ends the simulation having moved towards (or away) from the target simply by random chance. When the individual navigation weighting is very low $(w=0.01)$ then, as may be expected, the group navigational efficiency is generally low for all values of $\varepsilon$ and $k$ (Figure $2 \mathrm{a}$ ). When $w=1$, individuals in the group do not copy the movements of their neighbours, and hence no navigational information is shared ( $k$ has no effect). In this case, the group navigational efficiency is the same as the expected navigational efficiency for a non-social individual random walker moving with the same level of individual navigational error (Figure 2h).

At intermediate values of $w$ there is a non-linear relationship between the navigational efficiency, $E$, and the individual navigation weighting, $w$ (Figure 1). The plots in Figure 1a-f are qualitatively similar but show some subtle differences in the navigational efficiency, $E$, for particular combinations of $w$ and the number of influential neighbours, $k$, relative to the individual navigational error, $\varepsilon$. In particular, there is a clear peak in navigational efficiency for values of $w$ between 0.04 and approximately

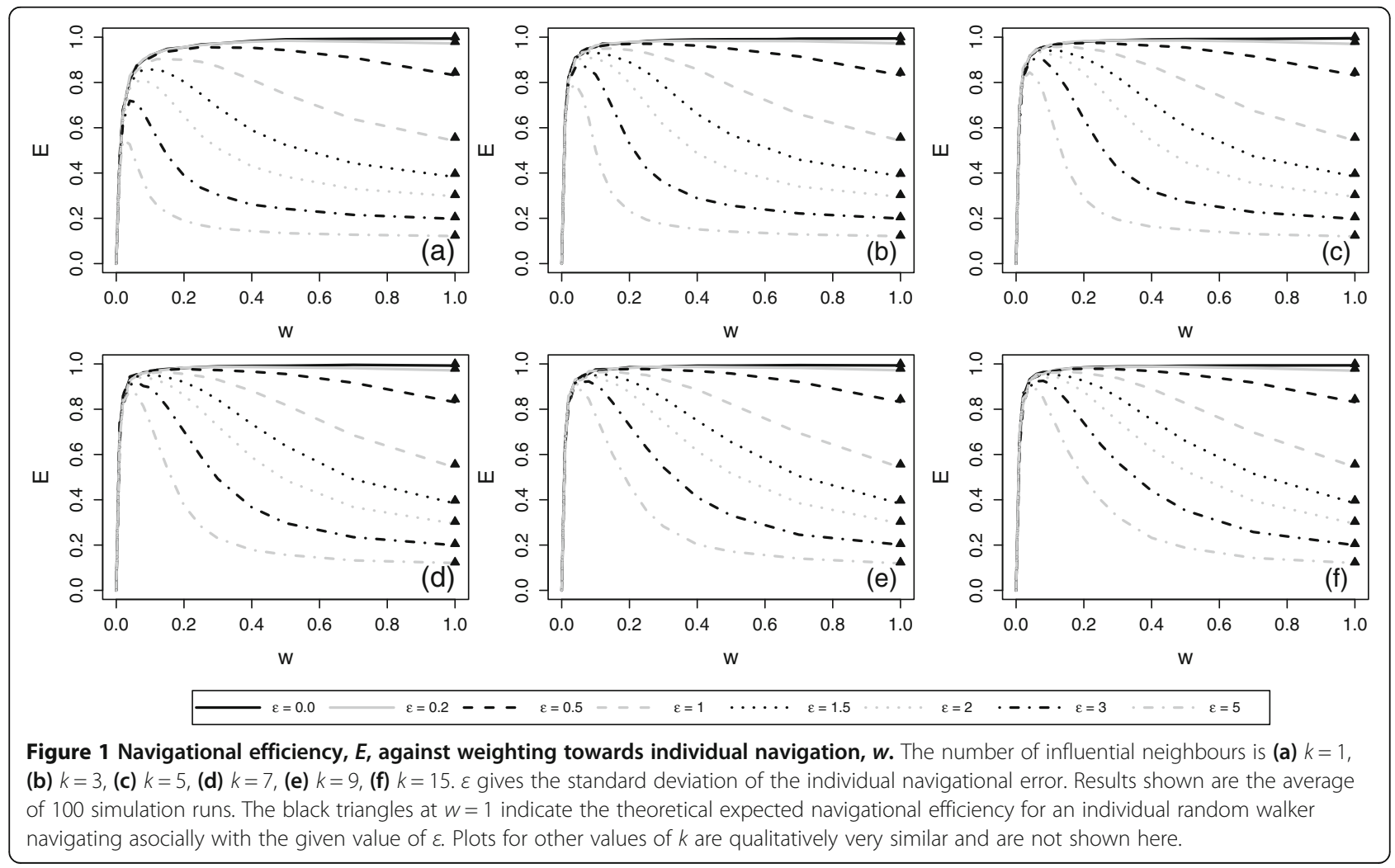



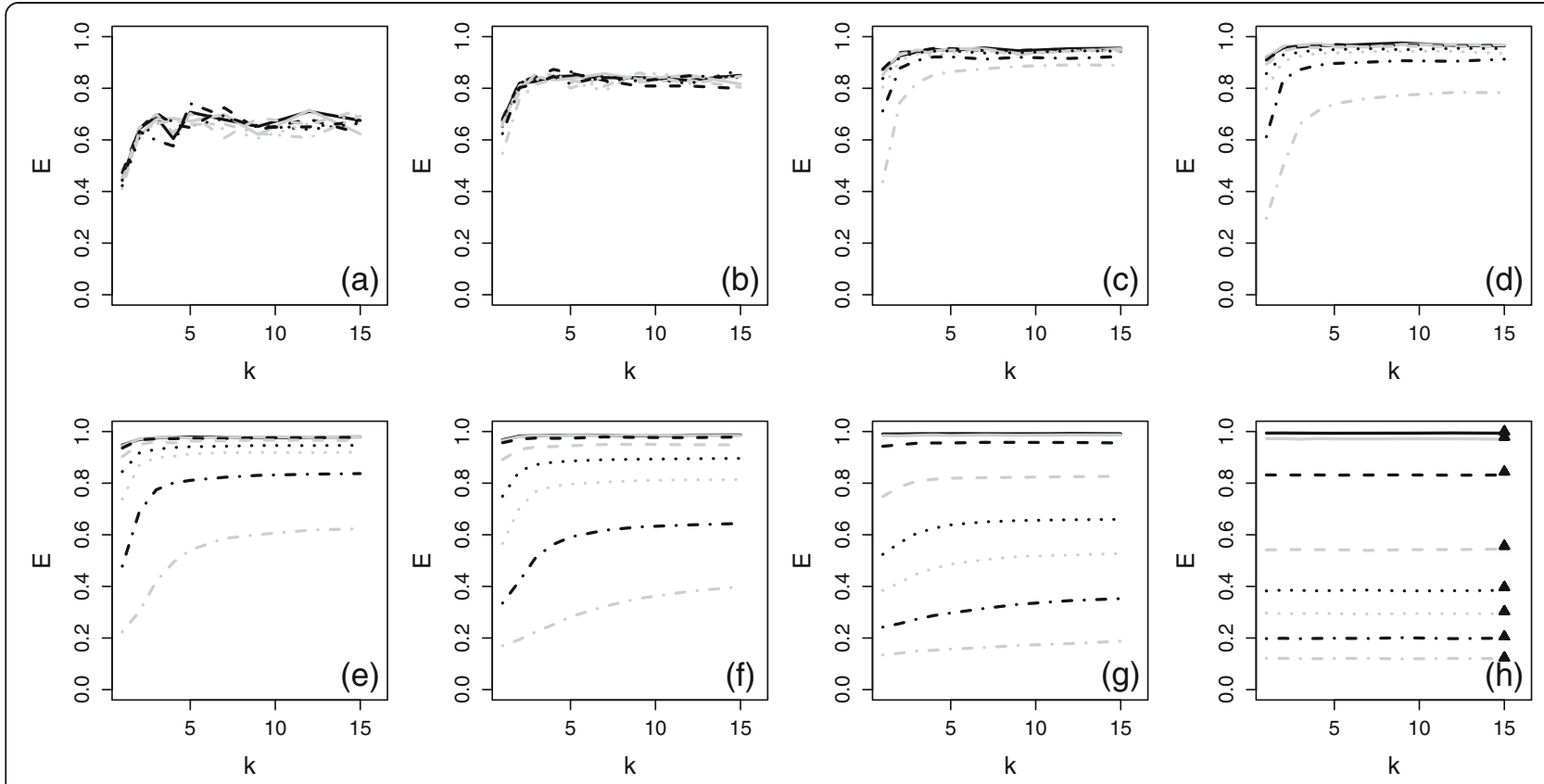

$$
\varepsilon=0.0
$$

$\varepsilon=0.2--\varepsilon=0.5$

$\varepsilon=1$

$\varepsilon=2 \quad \cdots-\cdot \varepsilon=3$

Figure 2 Navigational efficiency, $E$, against number of influential neighbours, $\boldsymbol{k}$. The weighting towards individual navigation is (a) $w=0.01$, (b) $w=0.02$, (c) $w=0.06$, (d) $w=0.1$, (e) $w=0.15$, (f) $w=0.25$, (g) $w=0.5$, (h) $w=1.0$. $\varepsilon$ gives the standard deviation of the individual navigational error. Results shown are the average of 100 simulation runs. The black triangles in (h) indicate the theoretical expected navigational efficiency for an individual random walker navigating asocially with the given value of $\varepsilon$.

0.25 , depending on $k$ and $\varepsilon$. Only in the case of $\varepsilon=0$ is this peak in navigational efficiency at an intermediate value of $w$ not evident (in such cases the navigational efficiency asymptotes at $w=1)$. As the individual navigational error increases ( $\varepsilon$ increases), the highest navigational efficiency occurs at a smaller value of $w$.

For a given value of the individual navigation weighting, $w$, there is a clear asymptotic relationship between $E$ and $k$ (Figure 2). Hence, for most values of $w$ and $\varepsilon$ there is very little relative navigational benefit in increasing $k$ much beyond 7. For example, the largest gain in navigational efficiency through increasing the number of influential neighbours above 7, across all values of $w$ and $\varepsilon$, is a $26 \%$ increase in efficiency when increasing from $k=7$ to $k=15$ for $w=0.3$ and $\varepsilon=5$ (plot omitted). In contrast if $k=7$ and $\varepsilon=5$, then changing from $\mathrm{w}=0.3$ to $\mathrm{w}=0.04$ or $\mathrm{w}=0.06$ leads to a $340 \%$ increase in navigational efficiency (Figure 1d). In other words, a much larger relative increase in navigational efficiency is gained by increasing the weighting given to copying the movement directions of a small subset of neighbours, rather than increasing the number of neighbours that are copied.

A general strategy for navigation in different environments The results in Figures 1 and 2 suggest that if the individual navigation error, $\varepsilon$, is low then there is little disadvantage in having a low value of the individual navigation weighting, $w$ (assuming $w \geq 0.02$, and depending on the number of influential neighbours, $k$ ), although a higher value of $w$ may give a very slightly higher navigation efficiency. However, for high $\varepsilon$ there is a clear disadvantage of having a larger value of $w$ (Figure 1). This suggests that there may be a small range of values for $w$ that are relatively efficient for all $\varepsilon$. This is particularly relevant for navigating animal groups that may be searching for targets where the level of individual navigation error differs for each target (e.g. because targets are at different distances, have different strengths of orientation cues or signals, etc.).

Our simulation results show that (in general), $0.02 \leq$ $w \leq 0.08$ give high levels of navigational efficiency across the widest range of values of $\varepsilon$, with smaller values of $w$ being more accurate at the largest individual navigation errors when the number of influential neighbours is lower. In particular, $w=0.06$ appears to give the best relative performance across the widest range of values of $k$ and $\varepsilon$ (Figure 3). When $\varepsilon \leq 0.5$, there are a few values of $w$ that give slightly better performances than $w=0.06$ (Figure 3), but the relative difference in efficiency is only ever marginal, and when the individual navigation error increases this relative difference is much greater going in the other direction. For example, $w=1$ is $14 \%$ more efficient than $w=0.06$, when $k=1$ and $\varepsilon=0$ (Figure 3a). 


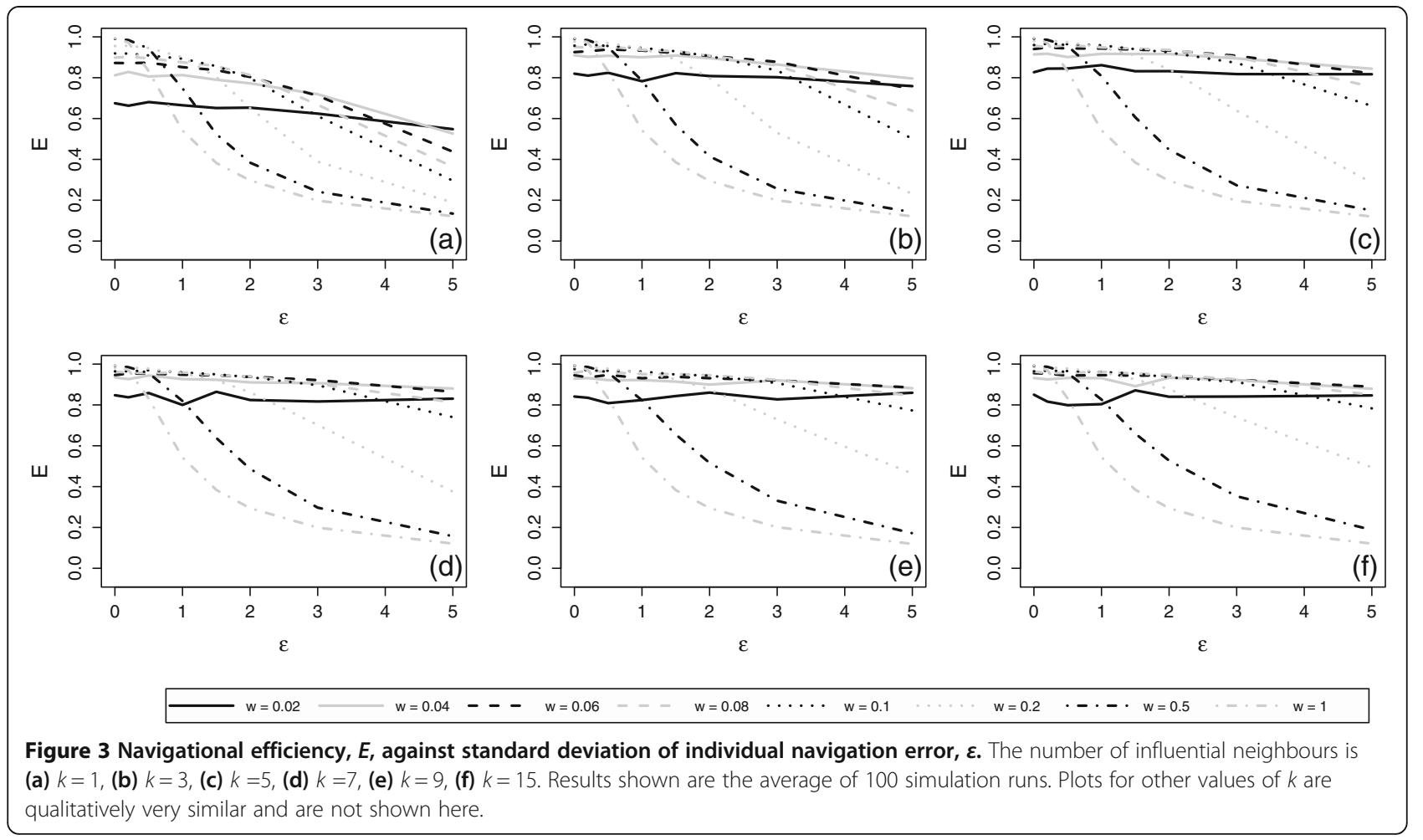

However, when $k=1$ and $\varepsilon=5, w=0.06$ is $359 \%$ more efficient than $w=1$.

\section{Variation in navigational performance}

Figure 4 gives the variation in navigational performance across simulation realisations, measured as the coefficient of variation $(\mathrm{CV}=$ standard deviation/mean $)$ of
$d_{T}$, the distance of the centre of mass of the group from the target after 500 time steps of the simulation. It should be stressed that the given $\mathrm{CV}$ measures the variation in navigational performance, not the actual navigational performance itself (hence a low CV could correspond to consistently poor or consistently good navigational performance).

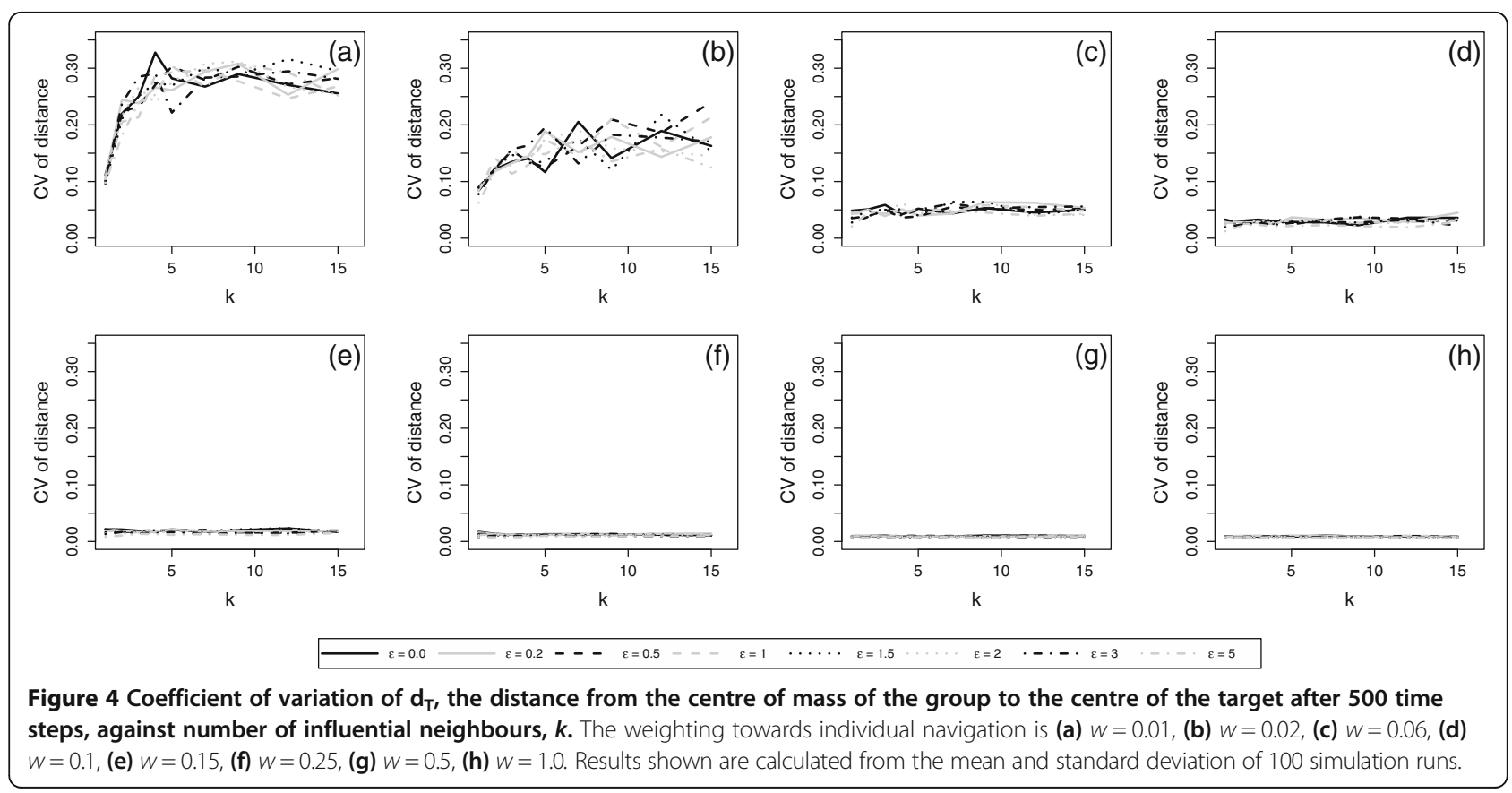


The variation in navigational performance is independent of $k$ (except for low values of $k$ and $w<0.04$, Figure $4 \mathrm{a}, \mathrm{b}$ ) and is also independent of the individual navigation error, $\varepsilon$. This shows that for a given level of individual navigation error, $\varepsilon$, and a given $w$, the navigational performance was on average stable across simulation runs. There is a clear trend in decreasing $\mathrm{CV}$ as $w$ increases. This is also clearly seen in Figure 2, where the data appears noisier for $w<0.04$ (Figure 2a, b), but much less noisy for high $w$ (Figure 2e-h). However, it should be stressed that the $\mathrm{CV}$ values observed are quite low, and hence simulation results can be considered stable for $w>0.04$ (Figure $4 \mathrm{c}-\mathrm{h}$ ). It is perhaps unsurprising that $w<0.04$ produces higher variation in navigational performance, since we know that $w=0$ will lead to entirely random movement by the group as a whole. One final point worth noting is that the value of $w$ leading to the best navigational efficiency, as discussed in a previous section, produces a slightly higher variation in navigational performance than higher values of $w$ (Figure 4c). Hence although the absolute CV values are still low in this case, this value of $w$ can perhaps be considered as a slightly more risky strategy (in that navigational performance is not as consistent) when compared to higher values of $w$.

\section{Effect of overall group size}

The simulation results discussed above were all completed for an overall group size of $N=40$ individuals (Table 1). To explore the effect of different group sizes, we completed additional simulations with a range of different group sizes, $N$ (Table 1; Figure 5), for the largest level of individual navigation error, $\varepsilon=5$ (i.e. the "worst case' for individual navigation that we consider). In all the cases illustrated in Figure $5(k=1, k=7, k=15, k=N)$, it is clear that while group navigation performance increases rapidly with group size for very small groups $(N<10)$, the performance then either asymptotes or decreases slightly at very large group sizes. This result matches the prediction made by Simons [5] that navigation performance using the "many wrongs principle" would reach a threshold level for large groups. Similarly, Codling et al. [7] demonstrated that a decrease in navigation performance at large group sizes can be explained through the effect of increased collision avoidance at high group densities. In general, Figure 5 illustrates that although there are some small quantitative differences in the results for very low overall group sizes ( $E$ is generally slightly lower), the qualitative trends, in terms of which parameter sets $(k$ and $w)$ produce the most accurate navigation, are the same as the results for $N=40$ (i.e. the best group navigation performance occurs when $k=7$ and $w=0.06$ ). Figure 5a shows that when $k=1$ and $\varepsilon=5$, group navigation performance is highest when $w=0.02$ (so that each neighbour will have greater influence on other individuals), but the overall group performance is much worse than when $k \geq 7$ for the same level of individual navigation error (Figures $2 \mathrm{~b}$ and $5 \mathrm{~b}, \mathrm{c}, \mathrm{d})$. In Figure $5 \mathrm{~d}$, we assume that $k=N$, so that each individual will copy the direction of movement of every other individual in the group. Although for large $N$, this may be beyond the cognitive abilities of most animals (also see Discussion), it serves to act as the extreme case of how individuals may copy others. Nevertheless, comparing Figure $5 \mathrm{~b}$ and $\mathrm{d}$, it is clear that there is no
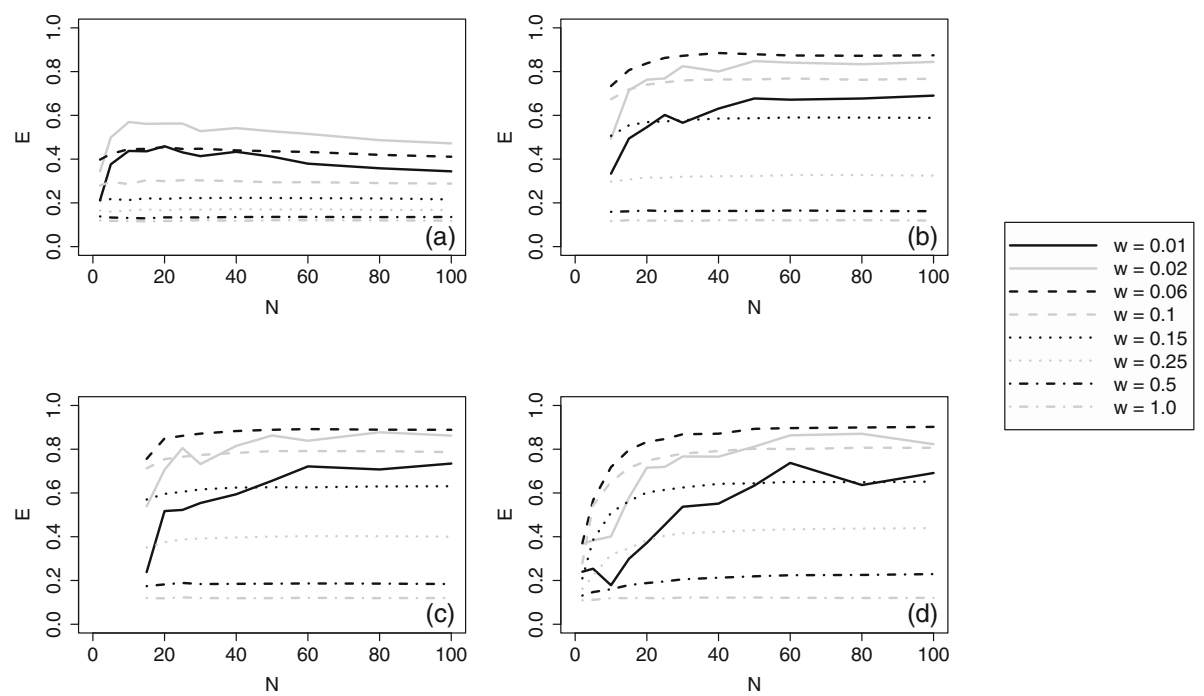

Figure 5 Navigational efficiency, $\boldsymbol{E}$, against overall group size, $\boldsymbol{N}$, for different values of $\boldsymbol{w}$. The number of influential neighbours is (a) $k=1$, (b) $k=7$, (c) $k=15$, (d) $k=N$. In (d), each individual responds to the direction of movement of every other individual in the group (regardless of group size). Results shown are the average of 100 simulation runs. 
significant relative benefit in having a large value of $k$ at large group sizes (when compared to $k=7$ or similar).

\section{Discussion}

We have used individual-based simulations to explore the information-sharing and copying dynamics of a leaderless social animal group navigating towards a fixed target. We assume that communication and informationsharing is indirect and arises through individuals partially copying the movement direction of their neighbours and weighting this information relative to their individual navigational knowledge of the target direction. We find that a highly efficient strategy in this simulation scenario, regardless of the level of individual navigational error, is for individuals to directly copy the most recent direction of movement of a small subset of their closest neighbours (e.g. $k=7)$ while only giving a small (e.g. $w=0.06)$ weighting to their own individual navigational knowledge.

It is clear that the mechanisms behind the many wrongs principle are more complex than assumed in [7], who only considered an arbitrary assumption of equal weighting $(w=0.5)$ between individual navigational knowledge and the movement directions of influential neighbours. Our results lead to the hypothesis that 'copying your neighbours' is a better general strategy than relying on your own navigational knowledge when navigating in groups (as long as individuals don't completely rely on others and contribute in a small way to the group decision-making). It is perhaps unsurprising that copying some navigational information from group neighbours is a sensible strategy. However, it is certainly surprising that the most efficient weighting given to individual navigation is so low ( $w=0.06$ or similar), particularly given that a very low weighting on individual navigation leads to poor navigation or even undirected movement $(w \leq 0.01)$. The basis behind giving a low weighting to individual navigation can be explained as follows. If your navigational ability is poor (and other group members are equally poor) then it is always better to copy your neighbours, as this allows the group to indirectly share information exactly as Simons [5] described ('the pooling of many inaccurate compasses'). Conversely, if your navigational ability is good (and other group members are equally good) then there is no disadvantage in copying your neighbours (although in such situations, individual navigation would be equally as efficient). If there is no disadvantage to copying your neighbours then this could provide a possible evolutionary basis for the many wrongs principle as an efficient navigation strategy [5,21-23]. Of course, this explanation is only valid if we assume the group is homogeneous and all individuals are equally poor (or good) at navigation. The model is likely to break down if some individuals in the group have different objectives e.g. [13], or if some individuals don't contribute any navigational knowledge or attempt to mislead others (although [3] demonstrated that uninformed individuals can improve consensus decision-making). An obvious extension of our current model would be to consider animal groups where the level of individual navigational error, and also the weighting given by other individuals to specific neighbours, could differ across the group. These additional factors could perhaps be explored in further simulation studies using evolutionary approaches e.g. [30]. Previous theoretical work has demonstrated that both the 'leader-follower' and the 'many-wrongs' model for group navigation can evolve in animal populations where individual fitness is obtained by balancing navigation success against costs of investment into navigation or social abilities $[22,23]$. In both studies the relative cost between investment in navigation and social abilities predominantly determines which model for group navigation is selected. We do not study possible evolutionary dynamics here and instead concentrate on conceptual mechanisms for information sharing within a group where there are no leaders present and all individuals have similar levels of navigational ability. Our model further differs from [22] and [23] since we explicitly consider the extent of social interactions (i.e. the number of influential neighbours, $k$ ).

It is worth considering how our model facilitates 'copying', an indirect form of information transfer within the group, and how this is best achieved. This can be considered as a cost/benefit trade-off between individual navigation and information gained from neighbouring group members. If no individual in the group contributes any navigational knowledge (i.e. if $w=0$ ) then the group will move as a non-oriented random walk and will typically never find the target (except by random chance). Conversely, if all individuals navigate independently (i.e. if $w \approx 1$ ) then no advantage is gained by being in a group (the 'many wrongs' does not apply). In our model, $k$ controls the level of influence of the rest of the group on each individual. This is why if $w$ is relatively large $(w>0.25)$, the group navigational efficiency is improved if $k$ is increased (Figure 2), as the group is able to have more influence on individual movement decisions. It is interesting to note that our simulation results showed that, although a higher value of $k$ gave typically higher navigational efficiency in an absolute sense, there was generally very little navigational improvement in a relative sense by increasing $k$ further than 7 influential individuals (i.e. the principle of diminishing returns). This is similar to the optimal number of influential neighbours suggested in other recent studies of collective animal behaviour [26-28]. In our simulations we did not include a 'cost' for increasing the number of influential neighbours that an individual can respond to and copy. However, it could be argued that larger values of $k$ are 
not biologically realistic since individuals are likely to have a cognitive limit to the information they can process, and the number of other group members they can respond to, in a short time [26]. More generally, it could be questioned whether animals interact with a fixed number of other group members. For example, empirical evidence on fish suggests alternative interaction ranges and mechanisms based on the visual system [31]. We therefore caution against a literal interpretation of the $k$-nearest neighbour interaction rule implemented in our model. The basic idea we want to capture with this interaction rule is that individuals can react to a larger or smaller fraction of the group. The specific interaction mechanisms in animals may well differ but our results suggest that while it is beneficial for animals to react to a small number of other group members, the added advantage of continuously reacting to large fractions of the group is negligible (Figure $5 \mathrm{~d}$ ).

In a study of animal taxis (individual navigation towards a target), Benhamou \& Bovet [24] considered the interplay between the 'tactic factor', $t$ (the weighting given to taxis / navigation) and persistence (the tendency for the animal to continue moving in the same direction as it did in the previous time step). In this model, the animal can be thought of as 'copying' information from its past movement to help in making decisions about where to move now (which parallels our model, where individuals copy or indirectly share information from other group members). In [24] the most efficient tactic factor across a range of individual navigational errors was $t=0.1$, and this compares very closely to the finding in our model that choosing $w=0.06$ is an efficient general strategy. Similarly, the shape of the non-linear curves in Figure 1 of [24] is almost exactly equivalent in a qualitative sense to our Figure 1. The fact that our results can be linked directly to this earlier study on purely individual navigation (taxis) hint at some interesting universal navigation principles about how to balance individual knowledge of the target with other available information. In particular, it would be interesting to combine our model with that of Benhamou \& Bovet [24] and explore the most efficient weighting between individual navigation, individual directional persistence, and copying movement directions of neighbours.

There are many assumptions in our simulation model that could be changed, and results should be considered in this context. As with [7], we haven't directly modelled blind regions e.g. $[6,32]$ and this could affect results, particularly when $k$ is large. We have assumed that individuals have navigation errors (that are independent of the distance from the target), but are able to accurately determine the movement directions of their neighbours or movement towards the centre of the group. We include an additional error, $\xi=0.1$, that corresponds to possible information processing errors or environmental turbulence, but a full exploration of the effect of increasing this error is beyond the scope of this study.

\section{Conclusions}

Our findings suggest that predominantly copying the movement direction of neighbours represents a successful group navigation strategy, irrespective of the level of individual navigation error. Interestingly, this balance between individual navigational information and social information (i.e. the movement of others) suggested by our model closely resembles the balance of taxis and persistence in individual navigation suggested by previous work [24]. Without explicitly considering cognitive limitations of individuals, our model suggests that it is not beneficial (in a relative sense) to individuals to copy more than approximately 7 neighbours.

Our simulation study is a conceptual exploration of the 'many wrongs' navigation problem. Such conceptual studies are important as they can provide useful theoretical insights into the dynamics and mechanisms that may underpin real animal movement. Our model has allowed us to create testable hypotheses about the most efficient way to navigate within a leaderless animal group, and these hypotheses could be tested using methods developed to analyse the movements of real animal groups $[33,34]$.

\section{Methods}

\section{Simulation structure \& virtual environment}

Our model is based on the discrete time group movement model in [7], which is a modified version of a well studied discrete time collective behaviour model e.g. $[6,32,35]$. Time steps and distances in the simulations are given in arbitrary units, have no physical meaning, and are used for comparative purposes only. In general, we assume a group size of $N=40$ individuals. Additional simulations completed show that the overall size of the group has little effect once a minimum viable group size is reached (Figure 5); it is the effect of influential neighbours that individuals copy that we are most interested in. Individuals are initially randomly distributed in a square of side length 100 units centred at $(x, y)=(0,0)$. All individuals in the group are initially aligned in the same direction, which is randomly chosen from a uniform circular distribution. The virtual two-dimensional environment is assumed to be homogenous except for a target site at $\left(x_{T}, y_{T}\right)=(0,1000)$. At each time step every individual in the group simultaneously updates its position according to hierarchical rules based on the location of the nearest neighbour(s) in the group in the previous time step. Individuals move with an average speed of 1 distance unit per time step and the simulations run for 500 time steps, and hence the theoretical maximum distance that the group should reach on average is 500 distance units away from the centre of the 
target (this is on average since fluctuations in speed can be introduced through a movement error term, see below). Consequently, we do not model movement within the local vicinity of the target and concentrate on the large scale navigation stage.

Given the structure of the virtual environment and the average movement speed of each individual, we define the navigational efficiency of the group as

$$
E=\left(1000-d_{T}\right) / 500,
$$

where $d_{T}$ is the distance from the centre of mass of the group to the centre of the target after 500 time steps of the simulation. Simulations were completed using the $\mathrm{R}$ language [36].

\section{Hierarchical rules of movement}

Models of the individual-level interactions in animal groups are usually based on a hierarchy of simple behavioural rules $[6,7,20,25,32,35]$ and we use a similar approach here. We assume each individual in the group has a radius of collision avoidance, $R_{C}$, and a radius of orientation interaction, $R_{O}$. At any given time step the movement behaviour of individual $i$ at position $\left(x_{i}, y_{i}\right)$ is dependent on the distance, $d$, between itself and its nearest neighbour $j$ at position $\left(x_{j}, y_{j}\right)$.

If $d<R_{C}$, then collision avoidance takes priority and individual $i$ will attempt to move directly away from individual $j$. The preferred movement direction is given by the unit vector

$$
\mathbf{r}=\frac{\left(x_{i}-x_{j}, y_{i}-y_{j}\right)}{\left\|x_{i}-x_{j}, y_{i}-y_{j}\right\|}
$$

If $R_{C}<d<R_{O}$, then navigation takes priority and individual $i$ will attempt to navigate towards the target based on the movement directions of its $k$ nearest neighbours and its own navigational knowledge. The preferred movement direction is given by the unit vector

$$
\mathbf{r}=\frac{w \mathbf{r}_{\text {ind }}+(1-w) \mathbf{r}_{g r p}}{\left\|w \mathbf{r}_{\text {ind }}+(1-w) \mathbf{r}_{g r p}\right\|}
$$

where $w$ is the weighting given to individual navigation.

Here $\mathbf{r}_{\text {ind }}$ represents the perceived movement direction from the individual's current position directly towards the target and is given by

$$
\mathbf{r}_{i n d}=\frac{\left(x_{T}-x_{i}, y_{T}-y_{i}\right)}{\left\|x_{T}-x_{i}, y_{T}-y_{i}\right\|}+\left(e r r_{x}, e r r_{y}\right)
$$

where $\left(x_{T}, y_{T}\right)$ is the centre of the target and $\operatorname{err}_{x} \sim N(0, \varepsilon)$ and $\operatorname{err}_{y} \sim N(0, \varepsilon)$ are normally distributed error terms, where the standard deviation, $\varepsilon$, is the parameter that determines the level of individual navigation error.
The group navigation component, $\mathbf{r}_{\text {grp }}$, is given by the unit vector average of the previous movement directions of the $k$ closest neighbours:

$$
\mathbf{r}_{\text {grp }}=\frac{\sum_{j=\mathbf{1}}^{k} \mathbf{v}_{j}}{\left\|\sum_{j=\mathbf{1}}^{k} \mathbf{v}_{j}\right\|}
$$

where $\mathbf{v}_{j}$ is the direction of movement of the $j$ th closest neighbour in the previous time step. In Eq. 5 , the $k$ closest neighbours of the focal individual are included, whether they are within $R_{O}$ of the focal individual or not. Larger values of $k$ therefore imply that individuals respond to a larger proportion of the group, while larger values of $w$ imply that the response of individuals to the movement of their influential neighbours is weaker.

If $d>R_{O}$, then group cohesion takes priority and individual $i$ will attempt to rejoin the group by moving directly towards the centre of mass of the group (the mean position of all individuals in the group). The preferred movement direction is given by the unit vector

$$
\mathbf{r}=\frac{\left(x_{C}-x_{i}, y_{C}-y_{i}\right)}{\left\|x_{C}-x_{i}, y_{C}-y_{i}\right\|}
$$

where $\left(x_{C}, y_{C}\right)=\frac{1}{N} \sum_{j=1}^{N}\left(x_{j}, y_{j}\right)$ is the centre of mass of the group at the end of the previous time step (calculated including the position of individual $i$ ).

Note that the model described here is a slight variation on the model of Codling et al. [7] who had an additional radius of cohesion outside which individuals were assumed to have left the group and would navigate and move independently. In addition we have not assumed any 'blind regions' as in some other collective movement models [6]. We assume that all individuals stay within sight of the rest of the group at all times. We choose values of $R_{C}=2$ and $R_{O}=15$ (Table 1 ) that are similar to [7] although this choice is arbitrary. Our aim is to use

Table 2 Expected navigational efficiency, $E$, for an individual asocial random walker navigating using the given standard deviation of individual navigation error, $\varepsilon$

\begin{tabular}{cc}
\hline $\begin{array}{c}\text { Standard deviation of individual } \\
\text { navigation error, } \boldsymbol{\varepsilon}\end{array}$ & $\begin{array}{c}\text { Expected navigational efficiency, } \\
\boldsymbol{E} \text { (3 decimal places) }\end{array}$ \\
\hline 0 & 1.00 \\
0.2 & 0.979 \\
0.5 & 0.843 \\
1 & 0.556 \\
1.5 & 0.397 \\
2 & 0.303 \\
3 & 0.205 \\
5 & 0.124 \\
\hline
\end{tabular}


values for the interaction radii that ensure globally polarised and cohesive group movement in the absence of navigation. The nature of these interaction rules, together with the fact that all individuals share a common target site, means that the group always maintains a high cohesion level (i.e. it is very unlikely that the group splits into sub-groups in this scenario).

\section{Theoretical navigational efficiency at the individual level} The navigational error term introduced in Eq. (4) can be linked to the standard definition of individual navigation efficiency, which is defined in terms of the average correlation of the direction of each realised move with the goal direction towards the target [24]. We randomly simulated the error on 100,000 independent random movement steps towards an arbitrary goal direction and calculated the average correlation towards this goal direction across each step, for each of the values of $\varepsilon$ given in Table 1. These numerically estimated values are given in Table 2 and can be used to predict the expected navigational efficiency for an individual asocial random walker ( $w=1$ in our simulations).

\section{Implementing moves}

We assume that individuals are subject to an additional noise/error term (corresponding to short-scale information processing or movement errors, or environmental turbulence) when they attempt to move in their chosen preferred direction. If the preferred movement direction is $\mathbf{r}$ (corresponding to either Eqs. (2), (3) or (6) depending on the nearest neighbour distance) then we calculate the actual movement direction as follows

$$
\mathbf{v}_{i}=\mathbf{r}+\left(m o v_{x}, m o v_{y}\right)
$$

where $\operatorname{mov}_{x} \sim N(0, \xi)$ and mov $_{y} \sim N(0, \xi)$ are normally distributed error terms, where the standard deviation, $\xi=0.1$, is fixed and represents movement errors due to shorttime-scale information processing errors or environmental turbulence. Finally, the new spatial position of individual $i$ is updated to be $\left(\mathrm{x}_{\mathrm{i}}^{\prime}, \mathrm{y}_{i}^{\prime}\right)=\left(\mathrm{x}_{i}, \mathrm{y}_{i}\right)+\mathbf{v}_{i}$ (and hence the speed of movement is variable due to the introduced movement error/noise). Note that the 'navigation error' is typically much higher than the 'movement error'. Table 1 lists the main parameter values used in the simulations.

\section{Ethical approval}

The work undertaken here was based entirely on computer simulations. Hence no formal ethical approval is required.

\section{Competing interests}

The authors declare that they have no competing interests.

\section{Authors' contributions}

EAC designed the study, wrote the simulation code and undertook simulations, interpreted the results, and wrote the majority of the paper. NWFB assisted with the design of the model, helped with interpretation of results, and contributed to writing the paper. Both authors read and approved the final manuscript.

\section{Acknowledgements}

NWFB was funded by an AXA Postdoctoral Fellowship award.

Received: 18 December 2013 Accepted: 19 May 2014

Published: 13 June 2014

\section{References}

1. Krause J, Ruxton GD: Living in groups. Oxford, UK: Oxford University Press; 2002.

2. Conradt L, List C: Group decisions in humans and animals: a survey. Proc R Soc B 2009, 364:719-742.

3. Couzin ID, loannou CC, Demirel G, Gross T, Torney CJ, Hartnett A, Conradt L, Levin SA, Leonard NE: Uninformed individuals promote democratic consensus in animal groups. Science 2011, 2011(332):1578-1580.

4. Conradt L: Models in animal collective decision-making: information uncertainty and conflicting preferences. Interface Focus 2012, 2:226-240.

5. Simons AM: Many wrongs: the advantage of group navigation. Trends Ecol Evol 2004, 19:453-455.

6. Couzin ID, Krause J, Franks NR, Levin SA: Effective leadership and decisionmaking in animal groups on the move. Nature 2005, 433:513-516.

7. Codling EA, Pitchford JW, Simpson SD: Group navigation and the "manywrongs principle" in models of animal movement. Ecology 2007, 88:1864-1870.

8. Bode NWF, Wood AJ, Franks DW: Social networks improve leaderless group navigation by facilitating long-distance communication. Current Zoology 2012, 58:329-341.

9. Bell WJ: Searching behaviour: the behavioural ecology of finding resources. London, UK: Chapman and Hall Ltd; 1991.

10. Berdahl A, Torney CJ, loannou CC, Faria JJ, Couzin ID: Emergent Sensing of Complex Environments by Mobile Animal Groups. Science 2013, 339:574-576.

11. Dyer JRG, loannou CC, Morrell $\amalg$, Croft DP, Couzin ID, Waters DA, Krause J: Consensus decision making in human crowds. Anim Behav 2008, 75:461-470.

12. Dyer JRG, Johansson A, Helbing D, Couzin ID, Krause J: Leadership, consensus decision making and collective behaviour in humans. Proc $R$ Soc B 2009, 364:781-789.

13. Conradt L, Roper TJ, Couzin ID, Krause J: "Leading according to need" in self-organizing groups. Am Nat 2009, 173:304-312.

14. Bergman $\mathrm{G}$, Donner $\mathrm{KO}$ : An analysis of the spring migration of the common scooter and the long tailed duck in southern Finland. Acta Zool Fenn 1964, 105:1-60.

15. Dell'Ariccia G, Dell'Omo G, Wolfer DP, Lipp HP: Flock flying improves pigeons' homing: GPS track analysis of individual flyers versus small groups. Anim Behav 2008, 76:1165-1172.

16. Faria JJ, Codling EA, Dyer JRG, Trillmich F, Krause J: Navigation in human crowds; testing the many-wrongs principle. Anim Behav 2009, 78:587-591.

17. Codling EA, Hill NA, Pitchford JW, Simpson SD: Random walk models for the movement and recruitment of reef fish larvae. Mar Ecol Prog Ser 2004, 279:215-224.

18. Simpson SD, Piercy JJB, King J, Codling EA: Modelling larval behaviour and population dynamics of coral reef fishes. Ecol Complex 2013, 16:68-76.

19. Grünbaum D: Schooling as a strategy for taxis in a noisy environment. Evol Ecol 1998, 12:503-522

20. Gregoire G, Chate H, Tu Y: Moving and staying together without a leader. Physica D 2003, 181:157-170.

21. Hancock PA, Milner-Gulland EJ, Keeling MJ: Modelling the many-wrongs principle: the navigational advantages of aggregation in nomadic foragers. J Theor Biol 2006, 240:302-310.

22. Guttal V, Couzin ID: Social interactions, information use, and the evolution of collective migration. Proc Natl Acad Sci 2010, 107:16172-16177.

23. Torney C, Levin SA, Couzin ID: Specialization and evolutionary branching within migratory populations. Proc Natl Acad Sci 2010, 107:20394-20399.

24. Benhamou S, Bovet P: Distinguishing between elementary orientation mechanisms by means of path analysis. Anim Behav 1992, 43:371-377. 
25. Viscido SV, Parrish JK, Grünbaum D: The effect of population size and number of influential neighbours on the emergent properties of fish schools. Ecol Model 2005, 183:347-363.

26. Ballerini M, Cabibbo N, Candelier R, Cavagna A, Cisbani E, Giardina I, Lecomte V, Orlandi A, Parisi G, Procaccini A, Viale M, Zdravkovic V: Interaction ruling animal collective behavior depends on topological rather than metric distance: Evidence from a field study. Proc Natl Acad Sci 2008, 105:1232-1237.

27. Bialek W, Cavagna A, Giardina I, Mora T, Silvestri E, Viale M, Walczak AM: Statistical mechanics for natural flocks of birds. Proc Natl Acad Sci 2012, 109:4786-4791.

28. Young GF, Scardovi L, Cavagna A, Giardina I, Leonard NE: Starling Flock Networks Manage Uncertainty in Consensus at Low Cost. PLoS Comput Biol 2013, 9:e1002894.

29. Codling EA, Plank MJ, Benhamou S: Random walk models in biology. $J$ R Soc Interface 2008, 5:813-834.

30. Wood AJ, Ackland GJ: Evolving the selfish herd: emergence of distinct aggregating strategies in an individual-based model. Proc R Soc B 2007, 274:1637-1642

31. Strandburg-Peshkin A, Twomey CR, Bode NWF, Kao AB, Katz Y, loannou CC, Rosenthal SB, Torney CJ, Wu HS, Levin SA, Couzin ID: Visual sensory networks and effective information transfer in animal groups. Curr Biol 2013, 23:R709-R711.

32. Couzin ID, Krause J, James R, Ruxton GD, Franks NR: Collective memory and spatial sorting in animal groups. J Theor Biol 2002, 218:1-11.

33. Bode NWF, Franks DW, Wood AJ, Piercy JJB, Croft DP, Codling EA: Distinguishing social from non-social navigation in moving animal groups. Am Nat 2012, 179:621-632

34. Del Mar DM, Penteriani V, Morales JM, Gurarie E, Ovaskainen O: A statistical framework for inferring the influence of conspecifics on movement behaviour. Methods Ecol Evol 2014, 5:183-189.

35. Aoki I: A simulation study on the schooling mechanism in fish. B Jpn Soc Sci Fish 1982, 48:1081-1088.

36. Development Core Team R: R: A language and environment for statistical computing. Vienna, Austria: R Foundation for Statistical Computing; 2012. ISBN 3-900051-07-0.

doi:10.1186/2051-3933-2-11

Cite this article as: Codling and Bode: Copycat dynamics in leaderless animal group navigation. Movement Ecology 2014 2:11.

\section{Submit your next manuscript to BioMed Central and take full advantage of:}

- Convenient online submission

- Thorough peer review

- No space constraints or color figure charges

- Immediate publication on acceptance

- Inclusion in PubMed, CAS, Scopus and Google Scholar

- Research which is freely available for redistribution 